\title{
Preserving Older Adults' Routine Outdoor Activities in Contrasting Neighborhood \\ Environments through a Physical Activity Intervention
}

Abby C. King ${ }^{1}$, Deborah $\mathrm{Salvo}^{2}$, Jorge A. Banda ${ }^{2}$, David K. Ahn², James E. Chapman ${ }^{3}$, Thomas

M. Gill ${ }^{4}$, Roger A. Fielding ${ }^{5}$, Jamehl Demons ${ }^{6}$, Catrine Tudor-Locke ${ }^{7,8}$, Andrea Rosso ${ }^{9}$, Marco

$$
\text { Pahor }^{10}, \& \text { Lawrence D. Frank }{ }^{3}
$$

\section{Word Count: Abstract $=250 ;$ Main Text $=3436$}

${ }^{1}$ Division of Epidemiology, Department of Health Research \& Policy, and the Stanford Prevention Research Center, Department of Medicine, Stanford University School of Medicine, Stanford, CA (king@stanford.edu).

${ }^{2}$ Stanford Prevention Research Center, Department of Medicine, Stanford University School of Medicine, Stanford, CA. (Dr. Salvo is now at the University of Texas Health Science Center at Houston School of Public Health, Austin, TX) (deborah.salvo@uth.tmc.edu; jorge.banda@stanford.edu; david.ahn@stanford.edu).

${ }^{3}$ Urban Design 4 Health, and Schools of Population and Public Health and Community and Regional Planning, University of British Columbia, Vancouver, British Columbia, Canada (ldfrank@ud4h.com; jchapman@ud4h.com).

${ }^{4}$ Department of Medicine, Yale University School of Medicine, New Haven, CT 06504 (Thomas.gill@yale.edu).

${ }^{5}$ Jean Mayer USDA Human Nutrition Research Center on Aging, Tufts University, Boston, MA 02111 (roger.fielding@ $@$ tufts.edu).

${ }^{6}$ Gerontology and Geriatric Medicine, Wake Forest School of Medicine, Winston-Salem, NC 27157 [jdemons@wakehealth.edu].

${ }^{7}$ Department of Kinesiology, University of Massachusetts, Amherst, MA 01003 (ctudorlocke@umass.edu).

${ }^{8}$ Pennington Biomedical Research Center, Baton Rouge, LA 70808

${ }^{9}$ Department of Epidemiology, University of Pittsburgh Graduate School of Public Health, Pittsburgh, PA, 15213 (alr143@ @itt.edu).

${ }^{10}$ Department of Aging and Geriatric Research, University of Florida, Gainesville, FL 32611 (mpahor@ufl.edu).

Corresponding Author: Abby C. King, PhD, Stanford University School of Medicine, 259 Campus Drive, HRP Redwood Building, Room T221, Stanford, CA 94305-5405; email: king@stanford.edu, telephone: 650-723-6254. 


\begin{abstract}
While neighborhood design can potentially influence routine outdoor physical activities (PA), little is known concerning its effects on such activities among older adults attempting to increase their PA levels. We evaluated the effects of living in neighborhoods differing in compactness on changes in routine outdoor activities (e.g., walking, gardening, yard work) among older adults at increased mobility disability risk participating in the LIFE-Pilot PA trial (2003-07; ages 70-89 years; from Dallas, TX, San Francisco Bay area, Pittsburgh, PA, and Winston-Salem, NC). Analyses were conducted on the 400 LIFE-Pilot participants randomized to a one-year endurance-plus-strengthening PA intervention or health education control that completed oneyear PA assessment (CHAMPS questionnaire). Outcomes of interest were exercise and leisure walking, walking for errands, and moderate-intensity gardening. Neighborhood compactness was assessed objectively using geographic information systems via a subsequent grant (2008-12). PA increased weekly exercise and leisure walking relative to control, irrespective of neighborhood compactness. However, walking for errands decreased significantly more in PA relative to control (net mean[SD] difference=16.2 minutes/week[7.7], $\mathrm{p}=.037$ ), particularly among those living in less compact neighborhoods (net mean[SD] difference=29.8[10.8] minutes/week, $\mathrm{p}=.006)$. PA participants living in less compact neighborhoods maintained or increased participation in gardening and yard work to a greater extent than controls (net mean[SD] difference $=29.3[10.8]$ minutes/week, $\mathrm{p}=.007)$. The results indicate that formal targeting of active transport as an adjunct to structured PA programs may be important to diminish potential compensatory responses in functionally impaired older adults. Structured endurance-plusstrengthening PA may help older adults maintain or increase such routine activities over time.
\end{abstract}

Trial Registration: clinicaltrials.gov Identifier $=$ NCT01072500

Keywords: aging, older adults, built environment, neighborhood design, compactness, walkability, physical activity, walking, gardening, residential density 


\section{INTRODUCTION}

The aging-associated chronic diseases and functional decline that are linked with inactive lifestyles are major public health challenges both in the U.S. and globally [1,2]. With the increased infirmities and reduced function that often accompany aging among inactive adults, mobility disability and accompanying loss of independence become major threats to a vital old age $[3,4]$. For instance, maintaining the ability to engage in routine activities around or near the home, such as walking for errands, housework, and yard work, is significantly linked with continued independent living — a highly valued state among many older adults [5]. In addition to its important impacts on chronic disease prevention and control, regular physical activity remains among the most powerful methods for maintaining day-to-day physical function and mobility into older age [6,7]. Regular participation in light- to moderate-intensity routine activities may also produce metabolic and other health-related benefits that, at least for some groups of less active older adults, may be similar to those obtained with more strenuous activities [8]. There is growing evidence of the health-enhancing benefits of such "lifestyle" activities for adults aged 65 years and older, particularly when engaged in regularly $[8,9]$. As a result, there is increasing emphasis on including a lifestyle lighter-intensity recommendation in national physical activity guidelines for older adults $[8,10]$.

Unfortunately, a significant proportion of older adults get insufficient levels of regular physical activity to optimally maintain levels of daily function. This is particularly true where local environments are less supportive of walking [11-13]. Walking and other routine activities (e.g., housework) remain important for older adults irrespective of the types of neighborhood environments in which they live (i.e., more vs. less compact). Other types of routine activities, such as outdoor yard work and gardening, may be particularly relevant for those older adults living in what has typically been labeled as "less compact" environments with lower residential density and fewer destinations, but larger yards. While outdoor activities such as walking and yard work can provide enhancements in wellbeing and quality of life for young and old alike [14], the potential benefits of such light to moderate outdoor activities may be particularly important for the health and functioning of older adults [15-19].

The Lifestyle Interventions and Independence for Elders Pilot (LIFE-P) Study of 70-89 year old sedentary adults at risk for mobility disability evaluated the 12-month effects of a program of aerobic plus strengthening exercise versus a health education control on aspects of lower- 
extremity physical function of particular relevance for the prevention of mobility disability [20]. The original study found that over the course of one year, scores on the SPPB and the 400-meter walk test were significantly improved in the physical activity relative to health education arm (p values < .001) [20]. Volume of moderate-intensity physical activity/week (consisting primarily of walking) increased significantly in the physical activity compared to health education arm [20].

The current investigation is based on additional funding obtained at the close of LIFE-P to investigate impacts of stable neighborhood design characteristics on participants' intervention success. We specifically explored whether the compactness of the neighborhood in which a participant lived moderated the effects of the physical activity program in relation to increases in walking as well as maintenance of moderate-intensity gardening and yard work activitiesimportant routine activities that have been linked observationally to such neighborhood characteristics [21, 22]. Neighborhood compactness was ascertained objectively using a validated urban design "walkability" index [23]. We postulated that the physical activity intervention would be sufficiently robust to increase 12-month exercise- or leisure-related walking levels irrespective of neighborhood compactness. In contrast, we believed that the effects of neighborhood compactness could be especially salient for maintaining or increasing more utilitarian outdoor activities such as walking for errands or accomplishing gardening and yard work, which contribute directly to independent living. With respect to walking for errands, we explored whether the physical activity intervention would be generally helpful in maintaining this type of utilitarian walking and whether level of neighborhood compactness would make a difference in this age group [12]. With respect to gardening and yard work, we explored whether the physical activity intervention would be helpful in maintaining such outdoor activities, particularly in less dense neighborhoods that typically allow for or require greater amounts of gardening and yard work activities.

\section{METHODS}

\section{Participants and Procedures}

LIFE-P was a multi-site randomized controlled single-blind trial which tested a physical activity intervention compared with a "healthy aging" education intervention (hereafter referred to as the health education arm) in sedentary older adults at risk for major mobility disability. The 
physical activity intervention targeted aerobic activities, which consisted primarily of brisk walking, as well as lower-extremity muscle strengthening, flexibility, and balance exercises. The intervention involved a combination of center-based (supervised) and home-based physical activity, with a goal of 150 minutes of walking/week. Details of the trial, the two interventions, and the major outcomes as well as the CONSORT flow chart and related information have been published elsewhere [20, 24]. Briefly, participants were recruited from four field centers (Cooper Institute in Dallas, TX, Stanford University, Palo Alto, CA, University of Pittsburgh, PA, and Wake Forest University, Winston-Salem, NC). Study eligibility criteria included the following: ages 70-89 years; <20 minutes per week spent in structured physical activity during the past month; having a Short Physical Performance Battery (SPPB) score $\leq 9$ out of 12 [25]; able to walk 400 meters in 15 minutes without sitting or use of an assistive device [20]; not planning to move out of the area for at least 9 months; and giving informed consent. Recruitment occurred primarily through community outreach, mass mailing, and media promotion. A total of 424 individuals were randomized, with 400 having 12-month physical activity data that allowed them to be included in the current investigation. The institutional review boards at all participating sites approved the study protocol. Written informed consent was obtained from all study participants.

\section{$\underline{\text { Measurements }}$}

Objectively determined neighborhood compactness. Neighborhood compactness, a major dimension of neighborhood design, was measured using a validated "walkability index" [23], with observation-specific measures of the environment, using 250 meter buffers around each participant's household. Although neighborhood walkability studies with other populations (e.g., adults or youth) have used larger buffer sizes (500-1000 meters) [26], after conducting a sensitivity analysis comparing strength of association and model fit with buffer sizes of 250, 500 and 1000 meters (data not shown), it was determined that a 250 meter buffer best captured the "neighborhood" exposure for this population subgroup of retired, older adults, which tend to have lower walking speeds than younger populations [27]. The GIS-based walkability index used to determine neighborhood compactness was a composite of four stable variables drawn from the urban planning literature $[12,23]$ : net residential density (ratio of residential units to the land area devoted to residential use); intersection density (connectivity of street network measured as the ratio of number of intersections with three or more legs to land area of the block group in 
acres); land-use mix (diversity of land use types per block group; normalized scores ranged from 0 to 1 , with 0 being single use and 1 indicating an even distribution of floor area across five uses - residential, retail, entertainment, office, institutional); and retail-to-floor area ratio (retail building square footage divided by retail land square footage). Publicly available geospatial datasets, including year 2000 Census Block Group tiger files, and shapefile data from local landuse and transportation departments, were used to compute the buffer-based walkability index.

Given that the term "walkability" originated from a focus on transportation patterns of largely working-age adults [28], it does not necessarily follow that the same relationships between neighborhood walkability and physical activity will occur in older, retired adults. Commensurate with this point, more inconsistencies in the walkability-physical activity relationship have been reported for older populations [29-31]. Therefore, an arguably more neutral term, i.e., neighborhood compactness, was applied.

Higher and lower neighborhood compactness were defined using study site-specific median splits of the walkability index, which is a zero centered, composite score of net residential density, intersection density, land-use mix, and retail-to-floor area ratio [12] (see Table 2 for sitespecific descriptive information). Neighborhoods falling within the lower half of each study site's walkability index median were designated as "less compact" (i.e., more suburban), while neighborhoods falling within the upper half of the walkability index were designated as "more compact" (i.e., more urban).

Walking. The Community Healthy Activities Model Program for Seniors (CHAMPS) questionnaire was used to assess self-reported walking for exercise and leisure (two items) and for errands (one item) [32]. The CHAMPS, which assesses weekly frequency and duration of various physical activities over the past four weeks, has suitable psychometric characteristics and has been shown to be sensitive to change in a range of adult and older adult populations [33]. These CHAMPS variables also have been linked with neighborhood compactness/walkability and related constructs in older adults [12]. The walking variables were chosen because the LIFEP physical activity intervention specifically targeted walking, and because these variables are most typically associated with built environment features [34].

Outdoor gardening and yard work. The CHAMPS questionnaire includes two items related to gardening and yard work, as follows: a) doing "heavy" gardening (such as spading or raking), and b) doing "light" gardening (such as watering plants). Given the focus of the LIFE-P physical 
activity program on promoting moderate-intensity activities, the first item describing more intensive forms of gardening was of specific interest in the current investigation. For descriptive purposes, we also evaluated any between-arm or neighborhood-specific changes in the light gardening activity.

While we did not expect (and did not find) neighborhood environment effects for indoor routine activities such as housework, we have included the housework item from the CHAMPS (i.e., sweeping, vacuuming) for descriptive purposes (see Table 1).

\section{$\underline{\text { Statistical Analysis }}$}

Mixed-effects regression models were used to estimate the main effects of the physical activity intervention (vs. health education) and neighborhood compactness (more vs. less compact) on the difference from baseline to 12 months of the two forms of walking (minutes/week of exercise and leisure walking, and minutes/week of walking for errands), and outdoor gardening and yard work (minutes/week). Standardized effect sizes were estimated (minutes per week at 12 months minus minutes per week at baseline/standard deviation). Additionally, log likelihood ratio tests were used to identify potential interactions between study arm and neighborhood design (more compact or less compact). If a significant interaction was identified, the conditional effect of physical activity vs. health education on the 12-month change in the dependent variable given each neighborhood design (i.e., more or less compact) was estimated. The models were adjusted for sociodemographic characteristics known to be associated with walking and physical activity levels [35], including educational attainment level (elementary school, high school, college, postgraduate education), sex, and race/ethnicity (White, Black, Hispanic, Other). Additionally, the models were adjusted for the effect of study site (cluster-variable: Dallas, TX, Pittsburgh, PA, San Francisco Peninsula, CA, Winston-Salem, $\mathrm{NC}$ ), and baseline gait speed during the 400m walk (continuous variable)--a marker for mobility impairment that can affect routine activity engagement [20]. Alpha was set at $\mathrm{p}<.05$ for determining main effects and at $\mathrm{p}<.20$ for identifying potential neighborhood compactness by intervention arm interactions for further exploration [36]. All analyses were performed using SAS 9.3 (SAS Institute Inc., Cary, N.C.).

\section{RESULTS}

\section{$\underline{\text { Participants }}$}


Objective GIS-derived environmental data were evaluated for the 400 participants (94\% of sample) who completed the 12-month CHAMPS questionnaire (physical activity arm: $\mathrm{n}=205$, 96.2\%; health education: $\mathrm{n}=195,92.4 \%$ ). Mean age was $76.7 \pm 4.2$ years; $69.5 \%$ were women; and $26.2 \%$ represented racial/ethnic minorities. The two arms had similar baseline characteristics ( $p$ values $\geq .13)$, and about half (48.5\%) of participants in each arm lived in more compact neighborhoods (see Table 1). Neighborhood compactness descriptive statistics by study site, based on the walkability index by Frank et al.[23], are provided in Table 2.

$\underline{\text { Neighborhood compactness as a potential moderator of physical activity intervention effects }}$

Exercise and leisure walking. As expected, the covariate-adjusted mixed regression analysis indicated a significant main effect for study arm favoring the physical activity intervention, which increased exercise and leisure walking across the 12-month intervention period by a mean of 64.6 [SD=14.0] minutes/week relative to health education $(\mathrm{p}<.0001$; (effect size $=0.41$, $\mathrm{CI}=0.21,0.60$ ). There was no significant neighborhood compactness-by-study arm interaction effect for this outcome $(\mathrm{p}=.52)$. Physical activity participants living in more compact neighborhoods increased exercise and leisure walking by a mean of 55.1 [SD=20.4] minutes/week relative to their health education counterparts $(\mathrm{p}=.007)(\mathrm{effect}$ size $=0.33, \mathrm{CI}=0.05,0.61)$; physical activity participants living in less compact neighborhoods increased this type of walking by $73.6[\mathrm{SD}=19.5]$ minutes/week relative to their health education counterparts $(\mathrm{p}=.0002)$ (effect size $=0.51, \mathrm{CI}=0.23,0.79)$. Health education participants also increased exercise and leisure walking, but by less than 33 minutes/week on average, regardless of neighborhood compactness.

Walking for errands. A potential neighborhood compactness-by-study arm interaction effect was found for this relationship $(\mathrm{p}=.07)$ (Figure 1). There was no significant between-arm effect of the intervention on walking for errands among participants living in more compact neighborhoods; both arms had mean 12-month decreases of approximately 22 [SD=104] minutes/week (between-arm difference=-1.8 [SD=11.1] minutes/week, $\mathrm{p}=.88)$ (effect size=-0.02, $\mathrm{CI}=-0.29,0.26)$. In contrast, physical activity participants living in less compact neighborhoods reported mean 12-month decreases in walking for errands relative to health education participants of 29.8 [SD=10.8] minutes/week, $\mathrm{p}=.006$ (effect size=-0.19, $\mathrm{CI}=-0.47,-0.09$ ), after adjusting for covariates. (Mean 12-month physical activity change: -15.4 [SD=102.1] minutes/week; mean health education change: 8.7 [SD=145.1] minutes/week). 
Moderate-intensity gardening and yard work. A potential interaction effect between neighborhood compactness and study arm was detected for this relationship ( $\mathrm{p}=.16)$ (Figure 2). In evaluating this potential effect further, we found no significant differences over time in the modest amounts of moderate-intensity gardening reported by participants living in more compact neighborhoods regardless of intervention assignment (mean $=7.5[\mathrm{SD}=11.2]$ minutes/week, $\mathrm{p}=.50$ ) (effect size $=0.10, \mathrm{CI}=-0.18,0.38$ ). In contrast, physical activity participants living in less compact neighborhoods generally maintained their reported 12-month levels of moderateintensity gardening relative to a decrease for health education (a mean difference of 29.3 [SD=10.7] minutes/week of moderate-intensity gardening was found between the two interventions) ( $\mathrm{p}=.007)$ (effect size $=0.27, \mathrm{CI}=0.03,0.55)$. The mean 12 -month change for physical activity was 2.2 [SD=104.5] minutes/week, and for health education was -27.6 [SD=111.0] minutes/week. All reported findings are adjusted for covariates.

\section{DISCUSSION}

This randomized controlled investigation explored the potential moderating effects of neighborhood compactness on the impacts of a physical activity intervention, designed specifically to increase discretionary-time (exercise and leisure-based) physical activity, on routine outdoor activities that are often important for older adults' independent living, in addition to contributing to health enhancement as people age [8]. Irrespective of neighborhood compactness, the physical activity intervention had the expected positive effects on exercise and leisure walking, similar to other interventions targeting walking in older adults [37]. However, in contrast to this positive impact, the physical activity intervention was associated with a general decrease in walking for errands (an average 16 minutes per week reduction relative to health education) across the 12-month intervention period.

When compared with health education, the decrease in walking for errands was particularly pronounced for those participants living in less compact neighborhoods (a net decrease in physical activity participants of about 30 minutes/week), where proximity to destinations often is more limited and walking for errands or transport more difficult [21]. In light of the recognized benefits of active transport for aging in place [38], what appears to be a potential compensatory reduction in active transport in those older adults participating in the physical activity intervention merits further investigation. Similar compensatory behaviors have been noted in 
other physical activity studies involving older adults and other populations [39, 40]. One hypothesis is that participants in the physical activity arm may have perceived success in achieving sufficient levels of physical activity via the intervention activities, and thus chose to reduce their activity levels for transportation. It is possible that more explicit targeting of such forms of routine physical activity in conjunction with the other forms of intervention-based walking may be useful in this more infirm older adult population. Future interventions could explicitly encourage participants to increase their levels of discretionary-time physical activity without decreasing their physical activities of other domains (e.g., for transport). This could be achieved by explicitly tracking levels of routine outdoor activities (e.g., transport-based physical activity), and making sure that those levels do not change substantially throughout the intervention period. Intervention strategies could also be expanded to include a component targeting increases in active travel. It also may be worthwhile to explore the small unexpected increase in walking for errands among participants living in less compact neighborhoods who were randomized to health education. (Physical activity was not discussed in that program.)

In contrast to these results, the physical activity intervention was positively linked with maintenance of moderate-intensity gardening and yard work activities in the less compact neighborhoods in which such outdoor activities are more prevalent. In contrast, those participants living in less compact neighborhoods who did not receive the physical activity intervention experienced an almost 30-minute per week reduction in moderate-intensity gardening and yard work activities over the 12-month period. Preserving such outdoor instrumental activities in those neighborhood contexts in which they are often required reflects a potentially important area for maintaining independent living as people age.

For working age populations, greater levels of the four components contributing to the neighborhood walkability index (used in this investigation to characterize neighborhood compactness) have been associated consistently with greater levels of pedestrian walking and bicycling activities [41]. While general associations between greater neighborhood walkability/compactness levels and greater levels of walking also have been found among older populations [12, 42], more inconsistencies in this relationship have been reported. For example, several randomized controlled trials evaluating physical activity interventions in older adults have found that persons living in less compact, more "suburban" neighborhoods reported more walking than persons living in more compact neighborhoods [29-31]. Relevant to this point, 
LIFE-P physical activity participants living in less compact neighborhoods had a 25 percent greater increase in exercise and leisure walking relative to their health education counterparts than those living in more compact neighborhoods.

$\underline{\text { Study Strengths and Limitation }}$

Among the strengths of the current investigation is its focus on older-aged adults already showing some functional limitations - a growing though less studied, highly vulnerable group with respect to subsequent mobility disability [7] for which participation in routine outdoor activities may be particularly important. The use of an objective determination of neighborhood compactness that targeted stable macro-scale features of the neighborhood environment also reflects a methodological strength, as does the randomized controlled study design and 12-month intervention length. While other studies have explored the relations between certain aspects of neighborhood design and one or more outdoor routine or instrumental activities among older adults, the vast majority of such studies have used observational designs [12]. Although participation in the routine activities under study was captured using primarily single- or dualitem questions on a self-report questionnaire, that questionnaire (the CHAMPS) was specifically developed and validated for older adults participating in physical activity interventions [32, 43].

Study limitations include the lack of an objective measure of physical activity (e.g., accelerometry), which could have provided additional insights related to the potential impacts of neighborhood design on participants' structured as well as routine forms of physical activity. The study also lacked relevant information on participants' perceptions of their neighborhoods' walkability — which can provide additional useful information in relation to physical activity [44, 45]. So too, the questions pertaining to gardening and yard work focused specifically on those activities occurring around the individual's home. It would have been useful to also gather information concerning communal or community gardening activities, in light of their potentially positive impacts on residents' sense of trust and community investment as well as control of community violence [46-48].

\section{CONCLUSION}

In summary, the results of this experimental investigation indicate that, among older adults attempting to increase their regular physical activity levels, those living in less compact neighborhoods may be at particular risk for compensatory reductions in certain routine activities 
such as active transport. In contrast, older adults from less compact neighborhoods may particularly benefit from such physical activity programs through maintaining their ability to engage in other routine activities such as gardening and yard work. The increasing evidence of the health-enhancing benefits of such "lifestyle" activities for older adults supports further systematic investigation of methods for maintaining or increasing these forms of regular physical activity, which are often ignored in community physical activity programming. In particular, finding ways to encourage continued active transport as well as other instrumental forms of activity across different types of neighborhoods may help to contribute to overall levels of physical activity and, by extension, health and vitality as people age. 


\section{Competing Interests}

The authors declare that they have no conflicts of interest. All authors read and approved the final manuscript.

\section{Authors' Contributions}

AK: Conceived of this paper's investigation and its study design, participated in selection of measurements, oversaw portions of data collection, contributed to analysis and interpretation of results, and took the lead in writing the manuscript.

DS: Contributed to the investigation design and methods, data analysis and interpretation of results, wrote portions of the manuscript, edited the manuscript, approved final version of manuscript.

JB: Contributed to the investigation design and methods, interpretation of results, edited the manuscript, approved final version for publication.

DA: Contributed to data analysis, interpretation of results, and development of tables and figures; edited the manuscript, approved final version of manuscript.

JC: Contributed to selection of measures, conducted portions of data collection, contributed to the interpretation of results, approved final version of manuscript.

TG: Contributed to the investigation design and methods, edited the manuscript, approved final version of manuscript.

RF: Contributed to the investigation design and methods, interpretation of results, edited the manuscript, approved final version of manuscript.

JD: Contributed to manuscript edits and approved final version of manuscript.

CTL: Contributed to manuscript edits and approved final version of manuscript.

AR: Contributed to manuscript edits and approved final version of manuscript.

MP: Obtained funding for the original LIFE-P grant and contributed to its investigation design and methods, participated in selection of measurements, oversaw portions of data collection, contributed to interpretation of results, critical review of the manuscript, approved final version of manuscript.

LF: Contributed to the investigation design and methods, selection of measures and oversight of portions of data collection, data analysis and interpretation of results, edited the manuscript, approved final version of manuscript. 


\section{Acknowledgements}

This investigation was supported by Public Health Service Grant \#5R01HL089694 from the National Heart, Lung, \& Blood Institute awarded to Dr. King. It was also supported by the Lifestyle Interventions and Independence for Elders Pilot Study, funded by a National Institutes of Health (NIH)/National Institute on Aging Cooperative Agreement (U01 AG22376), and was sponsored in part by the Intramural Research Program, National Institute on Aging, NIH. Dr. Gill is the recipient of an Academic Leadership Award (K07AG043587) from the National Institute on Aging. The research was partially supported by the Claude D. Pepper Older Americans Independence Centers at the University of Florida (1 P30 AG028740), Wake Forest University (1 P30 AG21332), Tufts University (1P30AG031679), University of Pittsburgh (P30 AG024827), and Yale University (P30AG021342) and the NIH/NCRR CTSA at Stanford University (UL1 RR025744), at University of Florida (U54RR025208), and at Yale University (UL1 TR000142). Tufts University investigators are also supported by the Boston Rehabilitation Outcomes Center (1R24HD065688-01A1), and Dr. Fielding is supported by the U.S. Department of Agriculture, under Agreement No. 58-1950-0-014. Any opinions, findings, conclusion, or recommendations expressed in this publication are those of the authors and do not necessarily reflect the view of the U.S. Dept. of Agriculture.

The above funding sources had no involvement in the described research, including research development, execution, analysis, interpretation of results, and write-up and submission of study results. 


\section{Tables and Figures}

Table 1. Descriptive statistics, for the total sample and by randomization arm

(Subjects participated from Dallas, TX, San Francisco Bay area, CA, Pittsburgh, PA, and

Winston-Salem, NC in LIFE-P Study, 2003-07).

Table 2. Descriptive statistics of neighborhood compactness, by study site

Figure 1. Change in weekly minutes of walking for errands, by neighborhood compactness and intervention arm

Figure 2. Change in weekly minutes of heavy gardening, by neighborhood compactness and intervention arm 


\section{References}

1. World Health Organization. Country profiles on nutrition, physical activity, and obesity in the 53 WHO European Region Member States: Methodology and summary. Copenhagen, Denmark: World Health Organization, 2013 ISBN 9789289050036.

2. Schoenborn CA, Adams PF. Health behaviors of adults: United States, 2005-2007. Data from the National Health Interview Survey. U.S. Department of Health and Human Services, 2010 Contract No.: DHHS Publication No. (PHS) 2010-1573.

3. King AC, Guralnik JM. Maximizing the potential of an aging population through shaping an active and vital old age. JAMA. 2010;304:1944-45.

4. Field MJ, Jette AM. The future of disability in America. Washington, D.C.: The National Academies Press, 2007.

5. Fried TR, Tinetti ME, Iannone L, O'Leary JR, Towle V, Van Ness PH. Health outcome prioritization as a tool for decision making among older persons with multiple chronic conditions. Arch Intern Med. 2011;171(20):1854-6. doi: 10.1001/archinternmed.2011.424. PubMed PMID: 21949032; PubMed Central PMCID: PMC4036681.

6. Hirvensalo M, Rantanen T, Heikkinen E. Mobility difficulties and physical activity as predictors of mortality and loss of independence in the community-living older population. $\mathbf{J}$ Am Geriatr Soc. 2000;48(5):493-8. Epub 2000/05/16. PubMed PMID: 10811541.

7. Pahor M, Guralnik JM, Ambrosius WT, Blair S, Bonds DE, Church TS, et al. Effect of structured physical activity on prevention of major mobility disability in older adults: The LIFE Study Randomized Clinical Trial. JAMA. 2014. doi: 10.1001/jama.2014.5616. PubMed PMID: 24866862.

8. Loprinzi PD, Lee H, Cardinal BJ. Evidence to support including lifestyle light-intensity recommendations in physical activity guidelines for older adults. Am J Health Promot. 2015;29(5):277-84. doi: 10.4278/ajhp.130709-QUAN-354. PubMed PMID: 24575724.

9. Buman MP, Hekler EB, Haskell WL, Pruitt L, Conway TL, Cain KL, et al. Objective lightintensity physical activity associations with rated health in older adults. Am J Epidemiol. 2010;172(10):1155-65. Epub 2010/09/17. doi: kwq249 [pii] 10.1093/aje/kwq249. PubMed PMID: 20843864.

10. Sparling PB, Howard BJ, Dunstan DW, Owen N. Recommendations for physical activity in older adults. Bmj. 2015;350:h100. doi: 10.1136/bmj.h100. PubMed PMID: 25608694. 
11. Satariano WA. The disabilities of aging--looking to the physical environment. Am J Public Health. 1997;87(3):331-2. PubMed PMID: 9096527.

12. King AC, Sallis JF, Frank LD, Saelens BE, Cain K, Conway TL, et al. Aging in neighborhoods differing in walkability and income: Associations with physical activity and obesity in older adults. Soc Sci Med. 2011;73:1525-33. Epub September 23, 2011. doi: 10.1016/j.scoscimed.2011.08.032. http://dx.doi.org/10.1016/j.socscimed.2011.08.032.

13. Shumway-Cook A, Patla AE, Stewart A, Ferrucci L, Ciol MA, Guralnik JM. Environmental demands associated with community mobility in older adults with and without mobility disabilities. Phys Ther. 2002;82(7):670-81. PubMed PMID: 12088464.

14. Hartig T, Mitchell R, de Vries S, Frumkin H. Nature and health. Annu Rev Public Health. 2014;35:207-28. doi: 10.1146/annurev-publhealth-032013-182443. PubMed PMID: 24387090.

15. Cunningham DA, Paterson DH, Himann JE, Rechnitzer PA. Determinants of independence in the elderly. Canadian Journal of Applied Physiology. 1993;18:243-54.

16. Carman JP. Re-creating 'the backyard' in senior communities. The Journal of Active Aging. 2006;5(1):74-5.

17. Wang D, MacMillan T. The benefits of gardening for older adults: A systematic review of the literature. Activities, Adaptation \& Aging. 2013;37(2):153-81. Epub 14 Jun 2013. doi: 10.1080/01924788.2013.784942.

18. Chen TY, Janke MC. Gardening as a potential activity to reduce falls in older adults. J Aging Phys Act. 2012;20(1):15-31. PubMed PMID: 22190117.

19. Kerr J, Sallis JF, Saelens BE, Cain KL, Conway TL, Frank LD, et al. Outdoor physical activity and self rated health in older adults living in two regions of the U.S. Int J Behav Nutr Phys Act. 2012;9:89. doi: 10.1186/1479-5868-9-89. PubMed PMID: 22846594; PubMed Central PMCID: PMC3464785.

20. Pahor M, Blair SN, Espeland M, Fielding R, Gill TM, Guralnik JM, et al. Effects of a physical activity intervention on measures of physical performance: Results of the lifestyle interventions and independence for Elders Pilot (LIFE-P) study. J Gerontol A Biol Sci Med Sci. 2006;61(11):1157-65. Epub 2006/12/15. doi: 61/11/1157 [pii]. PubMed PMID: 17167156. 
21. Frank LD, Engelke PO, Schmid TL. Health and community design: The impact of the built environment on physical activity. Washington, DC: Island; 2003.

22. Frank LD, Kerr J, Rosenberg D, King AC. Healthy aging and where you live: Community design relationships with physical activity and body weight in older Americans. J Phys Act Health. 2010;7(suppl 1):S82-S90.

23. Frank LD, Sallis JF, Saelens BE, Leary L, Cain K, Conway TL, et al. The development of a walkability index: Application to the Neighborhood Quality of Life Study. Br J Sports Med. 2009. Epub 2009/05/02. doi: bjsm.2009.058701 [pii] 10.1136/bjsm.2009.058701. PubMed PMID: 19406732.

24. Fielding RA, Rejeski WJ, Blair S, Church T, Espeland MA, Gill TM, et al. The Lifestyle Interventions and Independence for Elders Study: design and methods. J Gerontol A Biol Sci Med Sci. 2011;66(11):1226-37. Epub 2011 Aug 8.

25. Guralnik JM, Simonsick EM, Ferrucci L, Glynn RJ, Berkman LF, Blazer DG, et al. A short physical performance battery assessing lower extremity function: association with selfreported disability and prediction of mortality and nursing home admission. J Gerontol. 1994;49(2):M85-94. Epub 1994/03/01. PubMed PMID: 8126356.

26. Sallis JF, Saelens BE, Frank LD, Conway TL, Slymen DJ, Cain KL, et al. Neighborhood built environment and income: examining multiple health outcomes. Soc Sci Med. 2009;68(7):1285-93. Epub 2009/02/24. doi: S0277-9536(09)00031-8 [pii] 10.1016/j.socscimed.2009.01.017. PubMed PMID: 19232809.

27. Bohannon RW. Comfortable and maximum walking speed of adults aged 20-79 years: reference values and determinants. Age Ageing. 1997;26(1):15-9. Epub 1997/01/01. PubMed PMID: 9143432.

28. Frank LD, Sallis JF, Conway TL, Chapman J, Saelens BE, Bachman W. Multiple pathways from land use to health: Walkability associations with active transportation, body mass index, and air quality. J Am Planning Assoc. 2006; in press.

29. Sallis JF, King AC, Sirard JR, Albright CL. Perceived environmental predictors of physical activity over 6 months in adults: activity counseling trial. Health Psychol. 2007;26(6):701-9. Epub 2007/11/21. doi: 2007-16656-008 [pii] 10.1037/0278-6133.26.6.701. PubMed PMID: 18020842. 
30. King AC, Toobert D, Ahn D, Resnicow K, Coday M, Riebe D, et al. Perceived environments as physical activity correlates and moderators of interventions in five studies. Am J Health Promot. 2006;21:24-35.

31. Kerr J, Norman GJ, Adams MA, Ryan S, Frank L, Sallis JF, et al. Do neighborhood environments moderate the effect of physical activity lifestyle interventions in adults? Health Place. 2010;16(5):903-8. doi: 10.1016/j.healthplace.2010.05.002. PubMed PMID: 20510642; PubMed Central PMCID: PMC2918657.

32. Stewart AL, Mills KM, King AC, Haskell WL, Gillis D, Ritter PL. CHAMPS physical activity questionnaire for older adults: outcomes for interventions. Med Sci Sports Exerc. 2001;33(7):1126-41. PubMed PMID: 11445760.

33. Wilcox S, Dowda M, Griffin SF, Rheaume C, Ory MG, Leviton L, et al. Results of the first year of Active for Life: Translation of two evidence-based physical activity programs for older adults into community settings. Am J Public Health. 2006;96:1201-9.

34. Giles-Corti B, Timperio A, Bull F, Pikora T. Understanding physical activity environmental correlates: Increased specificity for ecological models. Exerc Sport Sci Rev. 2005;33(4):17581. PubMed PMID: 16239834.

35. Bauman AE, Reis RS, Sallis JF, Wells JC, Loos RJ, Martin BW, et al. Correlates of physical activity: why are some people physically active and others not? Lancet. 2012;380(9838):25871. doi: 10.1016/S0140-6736(12)60735-1. PubMed PMID: 22818938.

36. Pocock SJ. Further aspects of data analysis. Clinical trials: A practical approach. New York: John Wiley \& Sons; 2013. p. 213-5.

37. Merom D, Gebel K, Fahey P, Astell-Burt T, Voukelatos A, Rissel C, et al. Neighborhood walkability, fear and risk fo falling and response to walking promotion: The Easy Steps to Health 12-month randomized controlled trial. Preventive Medicine Reports. 2015;2:704-10.

38. Langlois JA, Keyl PM, Guralnik JM, Foley DJ, Marottoli RA, Wallace RB. Characteristics of older pedestrians who have difficulty crossing the street. American Journal of Public Health. 1997;87:393-7.

39. Goran MI, Poehlman ET. Endurance training does not enhance total energy expenditure in healthy elderly persons. American Journal of Physiology. 1992;263:E950-E7. 
40. Kempen KPG, Saris WHM, Westerterp KR. Energy balance during an 8-wk energyrestricted diet with and without exercise in obese women. American Journal of Clinical Nutrition. 1995;62:722-9.

41. Frank LD, Schmid TL, Sallis JF, Chapman J, Saelens BE. Linking objectively measured physical activity with objectively measured urban form: findings from SMARTRAQ. Am J Prev Med. 2005;28(2 Suppl 2):117-25. PubMed PMID: 15694519.

42. Clarke P, George LK. The role of the built environment in the disablement process. Am J Public Health. 2005;95(11):1933-9. PubMed PMID: 16195520.

43. Wilcox S, Dowda M, Leviton LC, Bartlett-Prescott J, Bazzarre T, Campbell-Voytal K, et al. Active for Life: final results from the translation of two physical activity programs. Am $\mathbf{J}$ Prev Med. 2008;35(4):340-51. Epub 2008/09/10. doi: S0749-3797(08)00605-3 [pii] 10.1016/j.amepre.2008.07.001. PubMed PMID: 18779028.

44. Kirtland KA, Porter DE, Addy CL, Neet MJ, Williams JE, Sharpe PA, et al. Environmental measures of physical activity supports: perception versus reality. Am J Prev Med. 2003;24(4):323-31. PubMed PMID: 12726870.

45. Kweon BS. Large-scale environmental knowledge: Investigating the relationship between self-reported and objectively measured physical environments. Environment \& Behavior 2006;38(1):72-91. doi: doi:10.1177/0013916505280092.

46. Jacob JA. Exercise and gardening programs as tools to reduce community violence. JAMA. 2015;314(14):1435-7. doi: 10.1001/jama2015.9002.

47. Zick CD, Smith KR, Kowaleski-Jones L, Uno C, Merrill BJ. Harvesting more than vegetables: the potential weight control benefits of community gardening. Am J Public Health. 2013;103(6):1110-5. doi: 10.2105/AJPH.2012.301009. PubMed PMID: 23597347; PubMed Central PMCID: PMC3698715.

48. van den Berg AE, van Winsum-Westra M, de Vries S, van Dillen SME. Allotment gardening and health: a comparative survey among allotment gardeners and their neighbors without an allotment. Environmental Health. 2010;9(74). doi: http://www.ehjournal.net/conent/9/1/74. 
Figure 1. Change in weekly minutes of walking for errands, by neighborhood design and intervention arm

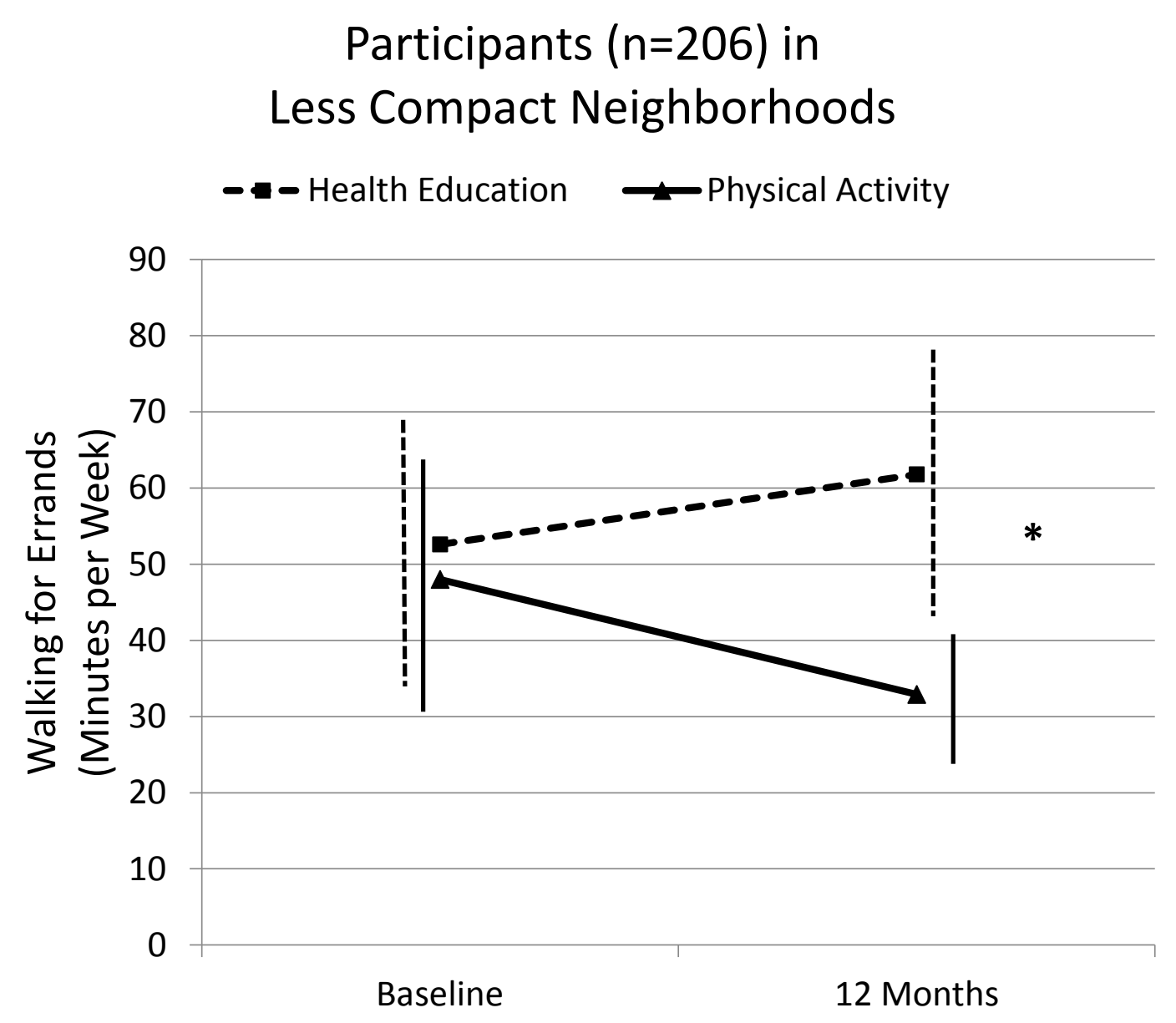

Bars indicate the $95 \%$ confidence interval.

* Between-arm difference significant at $p=.006$.

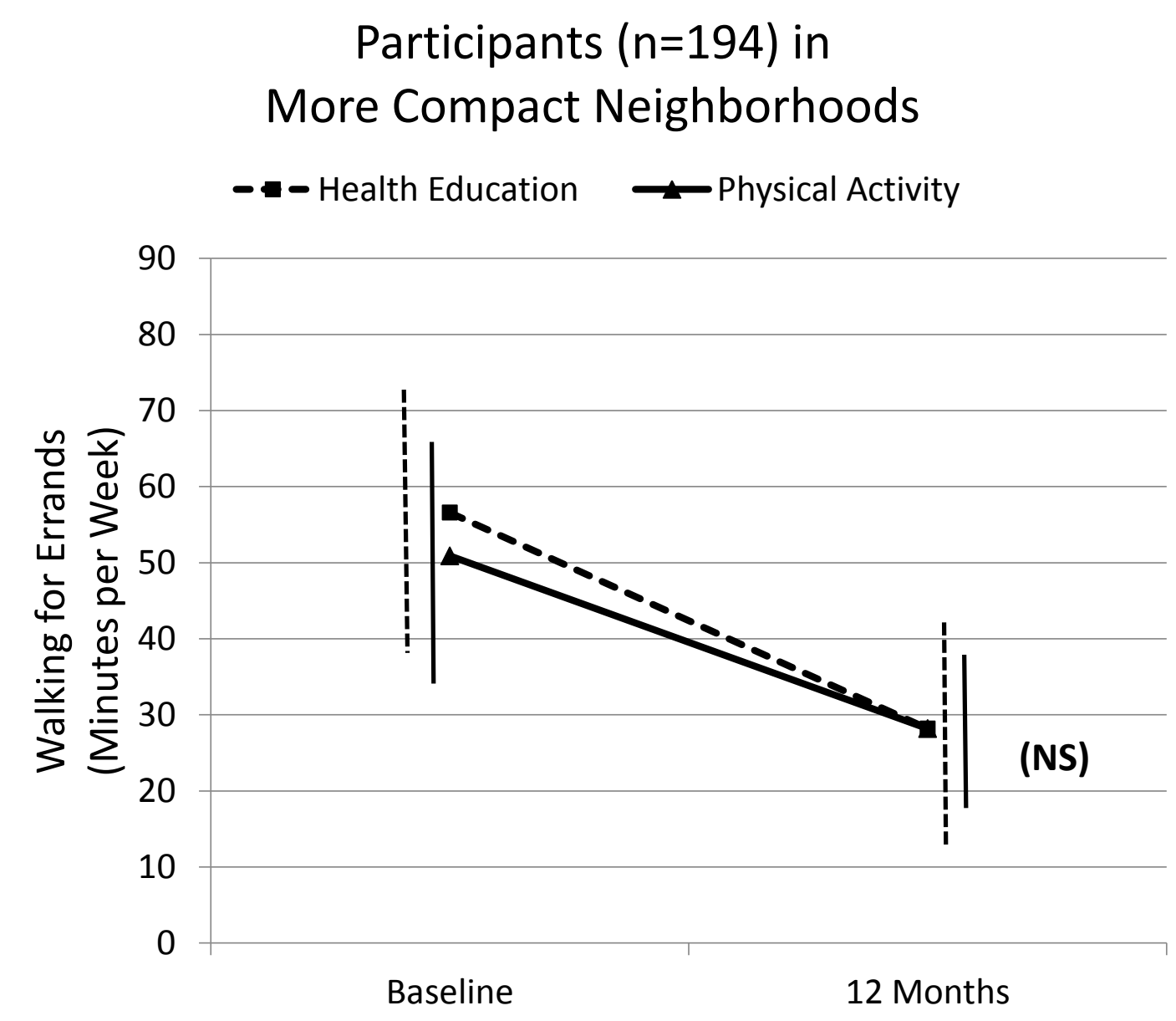

Bars indicate the $95 \%$ confidence interval. NS = Between-arm difference not significant. 
Figure 2. Change in weekly minutes of heavy gardening, by neighborhood design and intervention arm
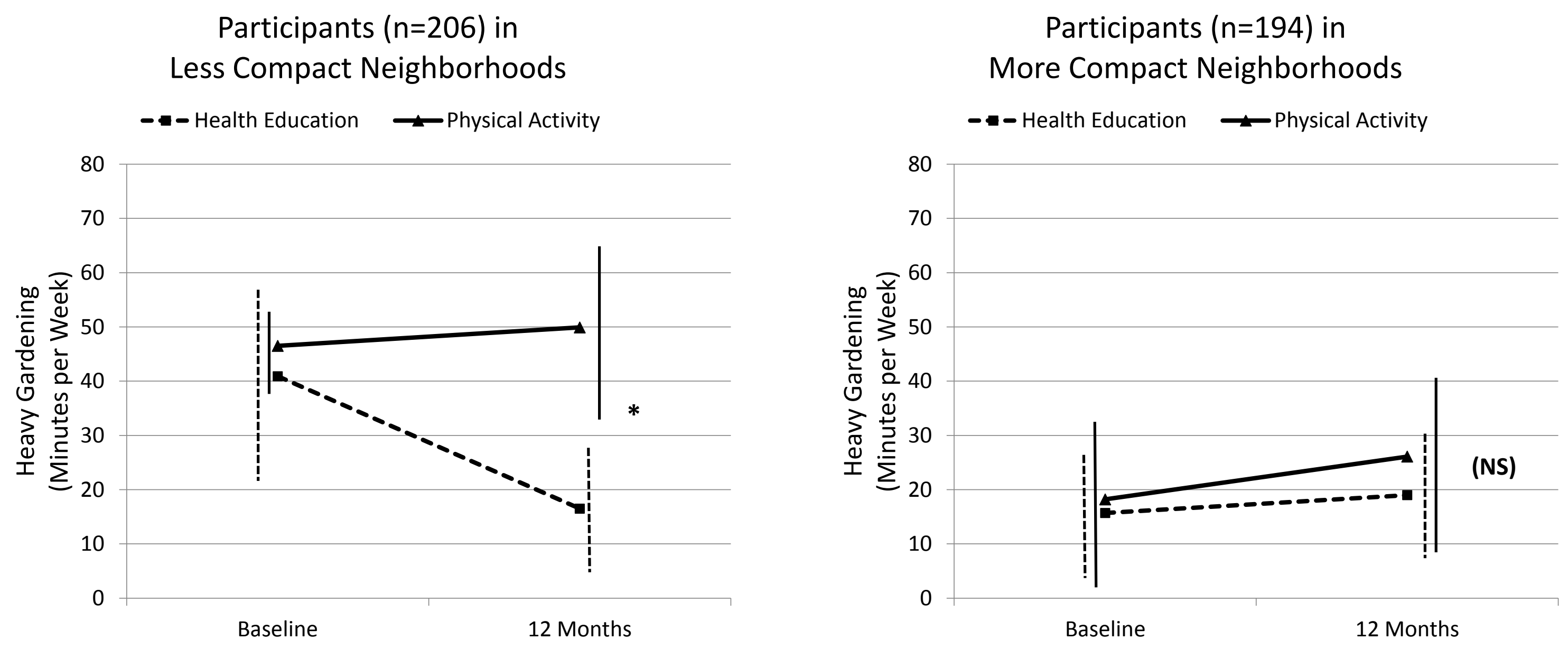

Bars indicate the $95 \%$ confidence interval.

* Between-arm difference significant at $p=.007$.

Bars indicate the 95\% confidence interval. NS = Between-arm difference not significant. 
Table 1. Descriptive statistics, for the total sample and by randomization arm

\begin{tabular}{|c|c|c|c|c|c|c|c|}
\hline \multirow[b]{2}{*}{ Variable (categorical) } & \multicolumn{2}{|c|}{ All } & \multicolumn{2}{|c|}{ Physıcal Activity } & \multicolumn{2}{|c|}{ Health Education } & \multirow[t]{2}{*}{ p value } \\
\hline & $\mathbf{N}$ & $\%$ & $\mathbf{N}$ & $\%$ & $\mathbf{N}$ & $\%$ & \\
\hline In more compact neighborhoods & 194 & 48.5 & 101 & 49.3 & 93 & 47.7 & .75 \\
\hline In less compact neighborhoods & 206 & 51.5 & 104 & 50.7 & 102 & 52.3 & \\
\hline \multicolumn{8}{|l|}{ Sex } \\
\hline Women & 278 & 69.5 & 142 & 69.3 & 136 & 69.7 & .92 \\
\hline Men & 122 & 30.5 & 63 & 30.7 & 59 & 30.3 & \\
\hline \multicolumn{8}{|l|}{ Race/Ethnicity } \\
\hline White & 295 & 73.8 & 153 & 74.6 & 142 & 72.8 & .87 \\
\hline Black & 73 & 18.2 & 36 & 17.6 & 37 & 19.0 & \\
\hline Hispanic & 20 & 5.0 & 10 & 4.9 & 10 & 5.1 & \\
\hline Other & 11 & 2.8 & 6 & 2.9 & 5 & 2.6 & \\
\hline Missing & 1 & 0.2 & 0 & 0.0 & 1 & 0.5 & \\
\hline \multicolumn{8}{|l|}{ Marital status } \\
\hline Married & 156 & 39.0 & 77 & 37.5 & 79 & 40.5 & .75 \\
\hline Separated/divorced & 61 & 15.0 & 36 & 17.6 & 25 & 12.8 & \\
\hline Widowed & 165 & 41.2 & 81 & 39.5 & 84 & 43.1 & \\
\hline Never married & 15 & 3.8 & 9 & 4.4 & 6 & 3.1 & \\
\hline Other & 3 & 0.8 & 2 & 1.0 & 1 & 0.5 & \\
\hline \multicolumn{8}{|l|}{ Education } \\
\hline Elementary & 15 & 3.8 & 7 & 3.4 & 8 & 4.1 & .44 \\
\hline High school & 116 & 29.0 & 58 & 28.3 & 58 & 29.7 & \\
\hline College & 187 & 46.8 & 103 & 50.2 & 84 & 43.1 & \\
\hline Postgraduate & 81 & 20.2 & 36 & 17.6 & 45 & 23.1 & \\
\hline Missing & 1 & 0.2 & 1 & 0.5 & 0 & 0.0 & \\
\hline \multicolumn{8}{|l|}{ Household income } \\
\hline$<\$ 5,000$ to $\$ 14,999$ & 63 & 15.8 & 37 & 18.1 & 26 & 13.3 & .51 \\
\hline$\$ 15,000$ to $\$ 34,999$ & 126 & 31.5 & 65 & 31.7 & 61 & 31.3 & \\
\hline$\$ 35,000$ to $\$ 74,999$ & 103 & 25.7 & 49 & 23.9 & 54 & 27.7 & \\
\hline$\$ 75,000$ or greater & 41 & 10.2 & 22 & 10.7 & 19 & 9.7 & \\
\hline Don't know, refused, missing & 67 & 16.8 & 32 & 15.6 & 35 & 18.0 & \\
\hline Variable (continuous) & Mean & SD & Mean & SD & Mean & SD & \\
\hline Age (yr.) & 76.7 & 4.2 & 76.6 & 4.2 & 76.9 & 4.2 & .45 \\
\hline Number in household & 1.8 & 0.9 & 1.7 & 0.9 & 1.8 & 0.9 & .81 \\
\hline Body Mass Index $\left(\mathrm{kg} / \mathrm{m}^{2}\right)$ & 30.3 & 6.1 & 30.7 & 6.2 & 29.8 & 5.9 & .13 \\
\hline Baseline SPPB score & 7.5 & 1.4 & 7.6 & 1.5 & 7.5 & 1.4 & .39 \\
\hline Walking gait speed (m/sec) & 0.9 & 0.2 & 0.9 & 0.2 & 0.9 & 0.2 & .72 \\
\hline \multicolumn{8}{|l|}{ CHAMPS Q - baseline (min/wk.): } \\
\hline Walking for exercise \& leisure & 68.0 & 97.3 & 61.1 & 91.1 & 75.2 & 103.2 & .15 \\
\hline Walking for errands & 50.7 & 94.5 & 49.8 & 92.5 & 51.6 & 96.7 & .85 \\
\hline Light gardening & 69.6 & 110.4 & 68.0 & 112.8 & 71.3 & 108.1 & .77 \\
\hline Heavy gardening & 32.0 & 93.5 & 32.8 & 92.2 & 31.2 & 95.1 & .87 \\
\hline Light housework & 184.5 & 180.2 & 187.5 & 180.5 & 181.3 & 180.2 & .73 \\
\hline Heavy housework & 25.5 & 90.0 & 30.9 & 100.0 & 19.8 & 78.1 & .22 \\
\hline
\end{tabular}

Between

group

value

Variable (categorical)

In less compact neighborhoods

Sex

Men

White

Hispanic

Other

Married

Separated/divorced

Never married

Othe

High school

$<\$ 5,000$ to $\$ 14,999$

$\$ 15,000$ to $\$ 34,999$

\$ $\$ 35,000$ to $\$ 7,999$

$\$ 75,000$ or greater

Don't know, refused, missing

Age (yr.)

Number in household

Body Mass Index $\left(\mathrm{kg} / \mathrm{m}^{2}\right.$

SPPB score

CHAMPS Q - baseline (min/wk.):

Walking for exercise \& leisure

\section{$\mathbf{N}$
194
206}

All 
Table 2. Descriptive statistics of neighborhood compactness by study site

$\begin{array}{lcccc}\text { Descriptive Statistic } & \text { Dallas, TX } & \text { Pittsburgh, PA } & \begin{array}{c}\text { San Francisco } \\ \text { Peninsula, CA }\end{array} & \text { Winston-Salem, NC } \\ \text { Median } & -0.9383 & 0.8619 & -0.1865 & -1.5056 \\ \text { Standard Deviation } & 1.7241 & 2.5143 & 1.9058 & 1.4557 \\ \text { Minimum } & -3.1530 & -3.0273 & -3.1622 & -3.2169 \\ \text { Maximum } & 8.9821 & 8.5953 & 6.7318 & 4.6827 \\ \text { Interquartile Range } & 1.3661 & 3.1964 & 2.0128 & 1.6567\end{array}$

NOTE: Neighborhood compactness was characterized using the "walkability index" developed by Frank et al (2010). The "walkability index" is a zero centered, composite score of net residential density, intersection density, land-use mix, and retail-to-floor area ratio. Low walkability index scores reflect less compact, single-use neighborhoods; while high walkability index scores reflect more compact, multi-use neighborhoods. 